\title{
Criminologie
}

\section{La déclaration de la victime au tribunal : évaluation de l'expérience du Palais de Justice de Montréal}

\section{Suzanne Laflamme-Cusson}

Volume 23, numéro 2, 1990

Après le crime : survivre

URI : https://id.erudit.org/iderudit/017295ar

DOI : https://doi.org/10.7202/017295ar

Aller au sommaire du numéro

Éditeur(s)

Les Presses de l'Université de Montréal

ISSN

0316-0041 (imprimé)

1492-1367 (numérique)

Découvrir la revue

Citer cet article

Laflamme-Cusson, S. (1990). La déclaration de la victime au tribunal : évaluation de l'expérience du Palais de Justice de Montréal. Criminologie, 23(2), 73-88. https://doi.org/10.7202/017295ar
Résumé de l'article

In October 1987, an experimental program was introduced in the Montreal Law Courts, inviting victims of crime and personal attack, as well as victims of burglary, to inform the Court of the consequences they suffered because of these acts; the program was called the Déclaration de la victime. An evaluative study of this experiment was initiated at the same time. The article presents some results : the response of the victims when offered the opportunity of taking part in the judicial debate, the content of the declarations, their use by the members of the Court, their possible influence on the proceedings and on the judicial decisions. 


\section{LA DÉCLARATION DE LA VICTIME AU TRIBUNAL: ÉVALUATION DE L'EXPÉRIENCE DU PALAIS DE JUSTICE DE MONTRÉAL Suzanne Laflamme-Cusson*}

In October 1987, an experimental program was introduced in the Montreal Law Courts, inviting victims of crime and personal attack, as well as victims of burglary, to inform the Court of the consequences they suffered because of these acts; the program was called the Déclaration de la victime. An evaluative study of this experiment was initiated at the same time. The article presents some results: the response of the victims when offered the opportunity of taking part in the judicial debate, the content of the declarations, their use by the members of the Court, their possible influence on the proceedings and on the judicial decisions.

\section{QU'EST-CE QUE LA DÉCLARATION DE LA VICTIME?}

La déclaration de la victime au tribunal est un moyen offert aux victimes d'actes criminels, dans les États de droit anglo-saxon, de participer au processus judiciaire. Dans ces États, à défaut de déclaration, le rôle des victimes se réduit à se soumettre, qu'elles le désirent ou non, à des assignations à comparaître comme témoins. En réponse au đésir d'un grand nombre de victimes, et dans le respect des principes de notre système de justice, un programme de déclaration de la victime propose aux personnes lésées de faire savoir à la cour, par le biais d'une déclaration, les conséquences physiques, psychologiques, sociales et financières qu'a eues sur elles l'acte criminel ou l'infraction subis. Cette participation est tout à fait volontaire car il n'est pas question ici d'ajouter au fardeau des victimes. La déclaration de la victime vise également l'accès à une meilleure justice pour tous en favorisant le prononcé de sentences plus éclairées. Elle a pris une multitude de formes selon les États ou les provinces où elle a été implantée.

\section{L'EXPÉRIENCE DU PALAIS DE JUSTICE DE MONTRÉAL}

Le projet montréalais a été élaboré par un comité de l'association québécoise Plaidoyer Victimes présidé par le juge Jean-Pierre Bonin. La mise

* Chercheure indépendante. 
en place et l'application du programme furent assumées par le ministère de la Justice du Québec — plus particulièrement par le Bureau d'aide aux victimes d'actes criminels - et sa coordination confiée à Me Esthel Gravel, substitut du procureur général. Une recherche évaluative concomitante, conçue et mise en œuvre par le professeur Micheline Baril, a été soutenue financièrement par le ministère de la Justice du Canada. Voyons les principales caractéristiques du projet pilote de déclaration de la victime au Palais de Justice de Montréal.

Toutes les victimes de crimes et infractions contre la personne et d'introductions par effraction ont été invitées à produire une déclaration de la victime lorsqu'un ou des suspects avaient été accusés relativement à leur affaire. Cette sélection a représenté plus de 8000 cas au cours de la période expérimentale de 18 mois, soit d'octobre 1987 à mars 1989.

Les victimes étaient approchées par la poste' ${ }^{\prime}$. On leur expédiait un questionnaire qui, s'il était complété et retourné au Palais de Justice, constituait leur déclaration. Les questions portaient sur quatre points: les blessures physiques, les séquelles psychologiques, les impacts sociaux et les pertes financières. Il y avait également un espace réservé à des commentaires libres. Ce questionnaire était accompagné d'une lettre d'explications sur l'usage qui pouvait en être fait en cour, comportant une invitation à s'adresser au Bureau de la déclaration pour obtenir de l'aide en cas de besoin, et d'une envelopperéponse au Bureau. Le tout était joint à la documentation expédiée par informatique aux victimes d'actes criminels dont les causes sont entendues en cour (INFOVAC) ${ }^{2}$. Les déclarations de victimes retournées au Bureau étaient insérées dans le dossier de la Couronne qui avait dès lors toute latitude pour les utiliser à son gré.

La recherche évaluative accompagnant ce projet pilote a porté sur de nombreux aspects de la déclaration, dont les principaux sont la réponse des victimes à l'invitation à produire une déclaration, le contenu qu'elles y ont mis, l'usage qui en fut fait par les professionnels de la justice (police, procureurs de la Couronne, avocats de la défense et juges), l'évaluation de l'expérience par ces derniers et par les victimes elles-mêmes, l'impact de la déclaration sur l'expérience des victimes dans le système judiciaire et enfin l'utilisation du Bureau de la déclaration de la victime par diverses catégories d'usagers.

1. Il arrivait également que des professionnels de divers organismes intéressés par le projet et ayant des formulaires sous la main en donnent aux victimes en les encourageant à les compléter.

2. Les proches de victimes d'homicides étaient pour leur part rejoints personnellement par la coordonnatrice de l'expérience et, s'ils se disaient intéressés, recevaient un formulaire remanié de déclaration. 
Ces aspects du projet pilote ont été analysés au moyen de plusieurs instruments ${ }^{3}$ : un cahier de contrôle de l'issue de chaque envoi de formulaire, un échantillon des déclarations elles-mêmes, un questionnaire aux procureurs sur l'usage fait à la cour de chacune des déclarations déposées au dossier de la Couronne, 30 entrevues avec les professionnels de la justice, deux questionnaires administrés à des échantillons de victimes (d'une part celles qui avaient complété une déclaration, d'autre part celles qui n'en avaient pas produit), des informations tirées des dossiers des accusés dans les affaires touchant les victimes échantillonnées, et enfin un relevé des activités du Bureau de la déclaration.

\section{QUELQUES RÉSULTATS DE LA RECHERCHE}

Parmi tous les résultats de la recherche, nous privilégierons ici ceux qui, à la fois, sont importants et ne requièrent pas de longues explications méthodologiques pour être bien compris.

\section{LA PARTICIPATION DES VICTIMES}

Sur les 8280 formulaires de déclaration qui furent expédiés, 364 sont revenus par la poste sans avoir atteint leur destinataire, 3619 sont revenus complétés tandis qu'on est demeuré sans nouvelles des 4297 derniers. Cela représente, pour les victimes présumées rejointes, un taux de réponse de $45,7 \%$. Pourquoj des victimes ont-elles répondu à l'invitation tandis que d'autres ne l'ont pas fait? La question fut posée à un échantillon de 247 victimes.

À ceux qui se souvenaient d'avoir produit une déclaration, on a proposé une série de motifs possibles et on leur a demandé dans quelle mesure chacun d'eux avait joué dans leur décision. Il en est ressorti qu'ils auraient complété la déclaration pour s'exprimer $(83,2 \%)$ et être entendus par la cour $(82,7 \%)$ et par l'accusé $(71,7 \%)$. Ensuite, ils auraient voulu avoir une influence sur la peine dans le sens de la sévérité $(62 \%)$ ou de la clémence $(2,8 \%)$, ou en faisant une proposition de sentence $(56 \%)$. Bon nombre de répondants $(37,3 \%)$ visaient entre autres un dédommagement, tandis que $35,7 \%$ se croyaient obligés de répondre à la sollicitation. Enfin, 3,5\% ont voulu retirer leur plainte par le biais de la déclaration.

Chez ceux qui se sont abstenus de compléter une déclaration, la raison la plus souvent retenue fut le désir d'oublier le crime $(46,2 \%)$. Ensuite, ils

3. Leur volume nous interdit de les reproduire ici. Nous trouvons néanmoins essentiel de reproduire en annexe le formulaire de déclaration en format réduit. 
ont craint la vengeance de l'accusé $(40,6 \%)$. Puis, par ordre d'importance, ils disent avoir considéré le crime pas assez grave $(34,9 \%)$, ou le questionnaire inadéquat $(31,1 \%)$, que cela n'aiderait pas leur cause $(30,2 \%)$, que c'était une perte de temps $(29,7 \%)$. Bon nombre auraient perdu ou oublié le questionnaire $(26,2 \%)$, n'avaient pas confiance dans le système judiciaire $(22,2 \%)$ ou ne voulaient pas nuire à l'accusé $(12,5 \%)$.

\section{LE CONTENU DES DÉCLARATIONS}

L'impression générale des personnes ayant eu l'occasion de lire un grand nombre de déclarations est celle d'une grande pudeur: sauf exception, les victimes parlent peu, elles répondent laconiquement aux questions qui leur sont posées et leurs commentaires sont également brefs. Elles seraient portées à minimiser leurs traumatismes physiques, psychologiques et sociaux. S'il y a parfois de l'exagération, ce serait, de l'avis des professionnels de la justice, au sujet des pertes financières, et encore, ce ne sont que des soupçons.

Notre analyse de contenu a porté sur un échantillon de 115 déclarations de victimes: 50 cas de voies de fait (dont 12 de violence familiale), 24 de vols qualifiés, 11 de menaces et 30 d'introductions par effraction. De ce nombre, $37(32,2 \%)$ ont mentionné des blessures physiques, dont $24(21 \%)$ ont eu recours à des soins médicaux, $57(49,6 \%)$ ont fait part de séquelles psychologiques, $76(66,1 \%)$, d'impact sur leur vie ou sur celle de leur entourage, $37(32,2 \%)$ de pertes financières directes et $52(45,2 \%)$ de pertes d'argent indirectes. Le montant déclaré des pertes directement attribuables au crime a varié de $12 \$ 10500 \$$, avec une médiane de 241 \$ tandis que celui des pertes indirectes s'est échelonné de $15 \$$ à $9055 \$$, avec une médiane de $200 \$$.

On a relevé des commentaires sur $67(58,2 \%)$ des déclarations. Dans 27 cas, ils faisaient mention de la sentence de l'accusé, le plus souvent en exprimant un souci de protection pour la victime ou pour autrui ( $16 \mathrm{cas}$ ). $\mathrm{Ou}$ bien la demande de protection était la seule exprimée ( 7 cas), ou bien elle s'accompagnait d'un souhait de clémence pour l'accusé (4), d'un traitement pour l'agresseur (3) ou d'une peine pour ce demier (2). Les autres suggestions relatives à la sentence concernaient une aide et un traitement pour l'accusé (5), une peine (2), l'équité (2), un dédommagement (1) ou autre chose (1). 


\section{L'UTILISATION DES DÉCLARATIONS EN COUR}

Cette analyse a porté sur 661 questionnaires remis à la Couronne avec la déclaration. Des 3500 questionnaires distribués ${ }^{4}$, 1525 ne furent pas retournés au Bureau, la raison majeure étant que la cause n'était pas terminée au terme de la période expérimentale; 721 ont été retournés non complétés pour divers motifs qu'il serait trop long de présenter ici; 593 n'ont pas été retenus pour l'étude parce qu'ils avaient un format qui s'est révélé inadéquat en cours d'étude et qu'une nouvelle version du questionnaire l'a remplacé. Voyons maintenant les résultats.

$44 \%$ des procureurs ont appris quelque chose de nouveau par le biais de la déclaration de la victime. Cela va à l'encontre de la crainte qu'avaient certains professionnels de la justice que la déclaration n'apporte pas d'information de plus que le rapport de police.

Une fois sur trois, le procureur a communiqué à la défense le contenu de la déclaration, le plus souvent — une fois sur quatre - lors des négociations préalables aux audiences.

Dans $10 \%$ des dossiers, le procureur signale que la déclaration l'a amené à assigner la victime comme témoin. L'inverse s'est produit également mais nous en ignorons la fréquence.

Une fois sur deux, comme le montre le graphique 1, le procureur a informé le juge du contenu de la déclaration. Dans la moitié de ces cas $(26,6 \%)$, il a déposé le document en preuve. Autrement, il s'est contenté de transmettre lui-même des renseignements qu'il y avait puisés, en précisant d'où il les tirait $(11,8 \%)$ ou non $(13,2 \%)$.

Lorsque la Couronne n'a pas utilisé la déclaration de la victime, ce fut le plus souvent $(36,9 \%)$ parce que les effets de la victimisation qu'on y trouvait étaient trop habituels pour qu'il y ait intérêt à les communiquer. Ce fut aussi en raison du témoignage antérieur de la victime $(25,5 \%)$, de l'absence de conséquences de l'acte criminel $(17,7 \%)$ et d'autres raisons pertinentes.

Dans le tiers des causes sur lesquelles porte notre analyse (233 sur 661), le procureur a eu le sentiment d'avoir fait une recommandation de sentence influencée — ou du moins supportée — par la déclaration de la victime. Une fois sur quatre, la seule influence a consisté à rassurer le procureur sur la pertinence de la recommandation envisagée de prime abord. Mais son influence

4. Des 3619 déclarations retournées au bureau, 119 ne furent pas remises à la Couronne: 108 sont arrivées trop tard, l'affaire étant déjà close du point de vue judiciaire, 7 se sont avérées d'une trop piètre qualité, une victime s'est désistée et nous manquons d'information pour les trois dernières. 
par le procureur de la couronne au moment de la sentence

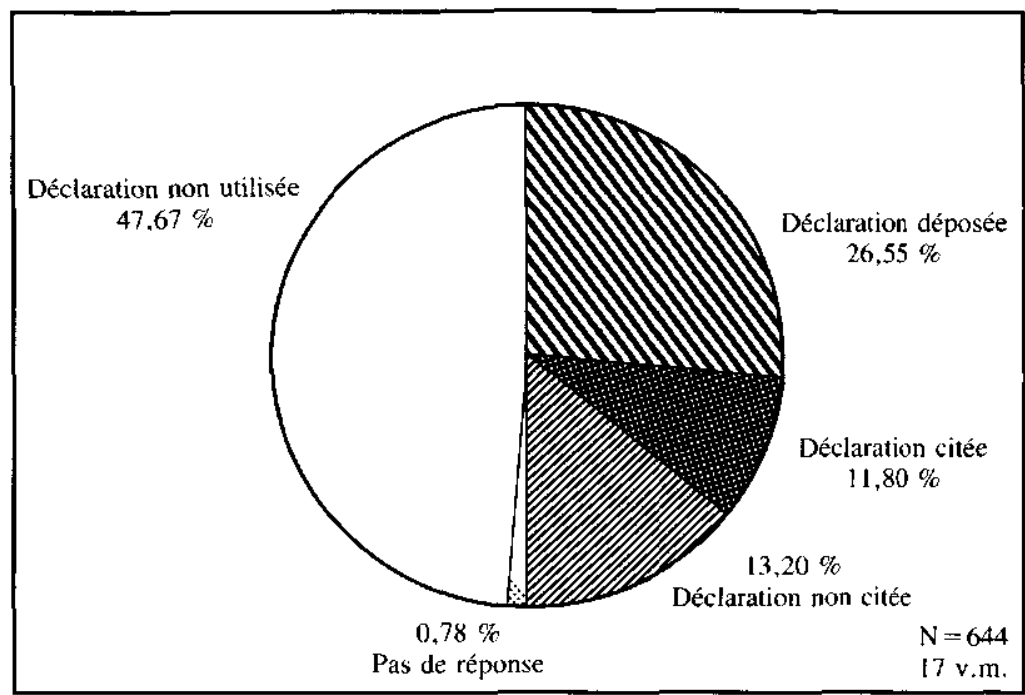

la plus fréquente s'est exercée sur le quantum de la peine proposée, également répartie en plus et moins sévère. Cela s'est fait de manière assez différente selon les infractions. C'est ainsi qu'il y eut, du fait de la déclaration, plus de clémence dans $17 \%$ des recommandations relatives à des voies de fait simples - ce que nous attribuons à la violence conjugale - et une sévérité accrue dans une proportion égale de voies de fait graves. Ce sont les deux types d'infractions où l'influence de la déclaration de la victime a été la plus marquée sur la recommandation de sentence des procureurs. Enfin la déclaration a eu peu d'influence sur la nature de la peine réclamée par la poursuite.

Selon la perception des procureurs, la défense a très rarement contesté le dépôt en preuve de l'écrit des victimes: à peine six contestations (trois ont réussi) sur 171 déclarations déposées. Quant aux juges, lors de la sentence, ils auraient mentionné dans 80 cas une information qui provenait de la déclaration de la victime. Ce nombre représente environ le quart $(23,7 \%)$ des causes où le procureur avait utilisé le document lors de ses représentations.

Toutefois, lorsque les juges mentionnent des éléments de la déclaration, ils ne leur attribuent pas forcément une influence sur leur décision. En effet dans $38 \%$ des cas, les juges ont parlé de choses qui provenaient de la déclaration, sans dire toutefois que celles-ci avaient joué sur le choix de la sentence, comme on le remarque au graphique 2 . 


\section{GRAPHIQUE 2}

L'impact de la déclaration sur les sentences tel qu'indiqué par les juges

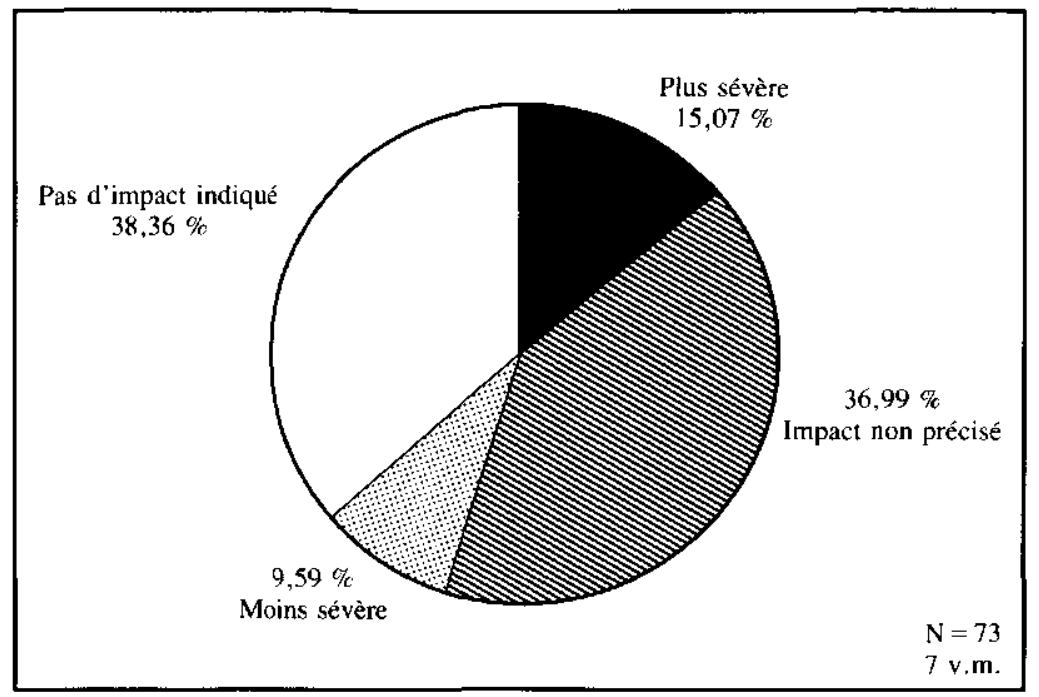

D'autre part, $37 \%$ auraient dit avoir été influencés sans préciser dans quel sens. Enfin, 15,1\% auraient explicité que leur sentence était plus sévère, contre $9,6 \%$, qu'elle était plus clémente. Étant donné toute la latitude laissée aux juges d'énoncer ou non les facteurs de modulation de leurs sentences, nos chiffres sur l'impact de la déclaration sur les sentences prononcées sont des minima.

\section{LES EFFETS DE LA DÉCLARATION SUR L'EXPÉRIENCE DES VICTIMES EN COUR}

Nous parlerons ici de l'implication plus ou moins active des victimes dans le processus judiciaire et de leurs impressions relatives au déroulement de leur affaire en cour. Les données de base de cette analyse sont des entrevues téléphoniques structurées, réalisées par la maison de sondange CROP, avec 126 répondants et 121 non-répondants à la déclaration de la victime. C'est donc la perception des victimes que nous rapportons ici.

Les trois quarts des personnes interviewées disent être allées à la cour. Qu'elles aient ou non rempli une déclaration ne fait pas de différence notable ${ }^{5}$. Lorsque les victimes ont eu l'occasion de s'entretenir avec le procureur

5. Nous qualifions de «notable» une différence de $10 \%$ et plus entre les groupes comparés. 
(39,7\% des cas), ce fut à l'initiative de ce dernier dans $84 \%$ des conversations avec les répondants à la déclaration contre seulement $62,5 \%$ des échanges avec les non-répondants. La différence est statistiquement significative $(\mathrm{p}<.05)$. Ces entretiens ont porté sur les conséquences du crime dans $60,4 \%$ des cas où la victime avait rempli une déclaration comparativement à 45,5\% lorsqu'elle n'en avait pas rempli (statistiquement non significatif). Les différences observées tendraient à appuyer un fait mentionné par des procureurs, à savoir que le contenu des déclarations les poussait parfois à prendre contact avec les victimes pour en savoir davantage ou pour suivre l'évolution dans le temps des situations décrites par les victimes.

Qu'elles aient ou non complété une déclaration, seule une partie des victimes ira témoigner en cour sur les faits de la cause ou sur l'impact des actes subis. Le graphique 3 compare, pour les deux échantillons, les filtres successifs entre la victimisation et le témoignage sur l'impact du crime.

\section{GRAPHIQUE 3}

Le filtrage de la cour entre la victimisation et le témoignage sur l'impact du crime

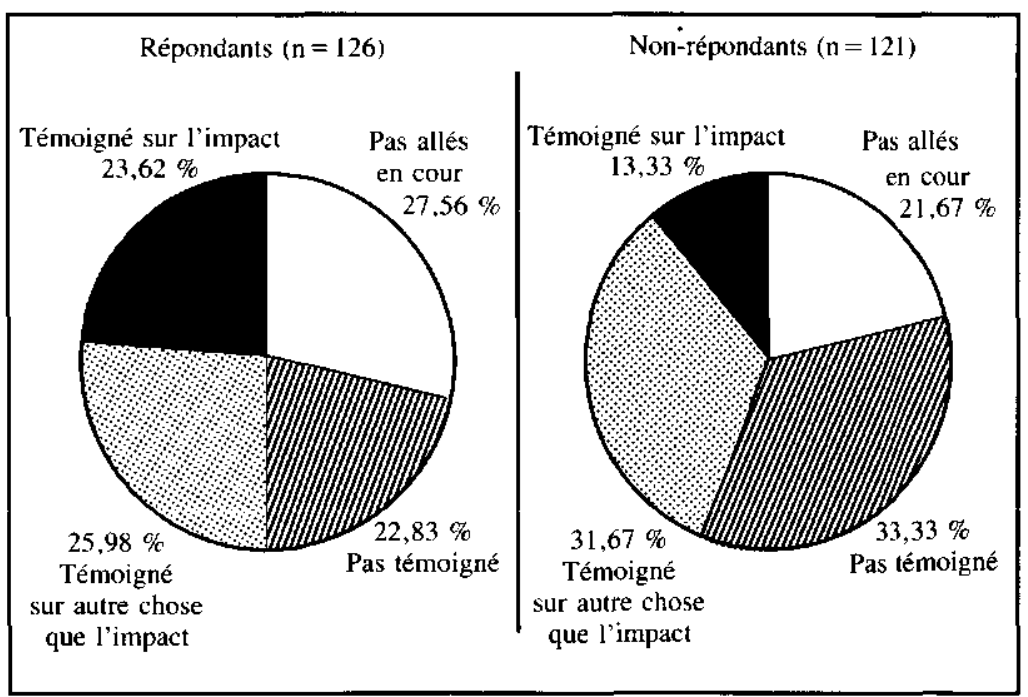

On constate que si les sujets ayant répondu à la déclaration sont allés presque aussi souvent en cour que les autres, ils y sont surtout allés moins souvent pour rien, c'est à dire sans être appelés comme témoins (22,8\% contre $33,3 \%)$. Par ailleurs, ils se sont plus fréquemment exprimés sur les conséquences des crimes subis $(23,6 \%$ comparé à $13,3 \%)$. 
Le fait d'avoir complété ou non une déclaration de la victime a-t-il un effet sur la satisfaction tirée de son expérience avec le système de justice? Apparemment pas, comme le montre le graphique 4 .

À l'évidence, les deux groupes sont également satisfaits. Faut-il en conclure qu'un programme de déclaration de la victime n'améliore pas le sort des victimes devant les tribunaux ou du moins leur contentement? Absolument pas. Pour en arriver à une telle conclusion, il aurait fallu comparer un groupe de personnes invitées à produire une déclaration à un groupe qui n'y aurait pas été invité, ce qui n'est pas notre cas. Dans le projet pilote du Palais de Justice de Montréal, toutes les victimes de certains types de crimes ont été approchées. Elles ont choisi d'y répondre ou pas: celles qui le voulaient se sont exprimées dans la déclaration tandis que les autres s'en sont abstenues.

Compte tenu de la piètre opinion des victimes à l'égard des tribunaux, telle qu'on la décèle d'un sondage à l'autre, le niveau de satisfaction des victimes rejointes par le programme est remarquablement élevé. Il serait néanmoins prématuré et peu scientifique de l'attribuer à l'expérience de déclaration de la victime.

\section{LES OPINIONS DES VICTIMES SUR LA DÉCLARATION}

C'est par le biais des réponses au sondage réalisé par CROP auprès des victimes que nous saisirons leurs opinions. Soulignons d'abord que 13 des

\section{GRAPHIQUE 4}

La satisfaction tirée de l'expérience avec le sistème judiciaire

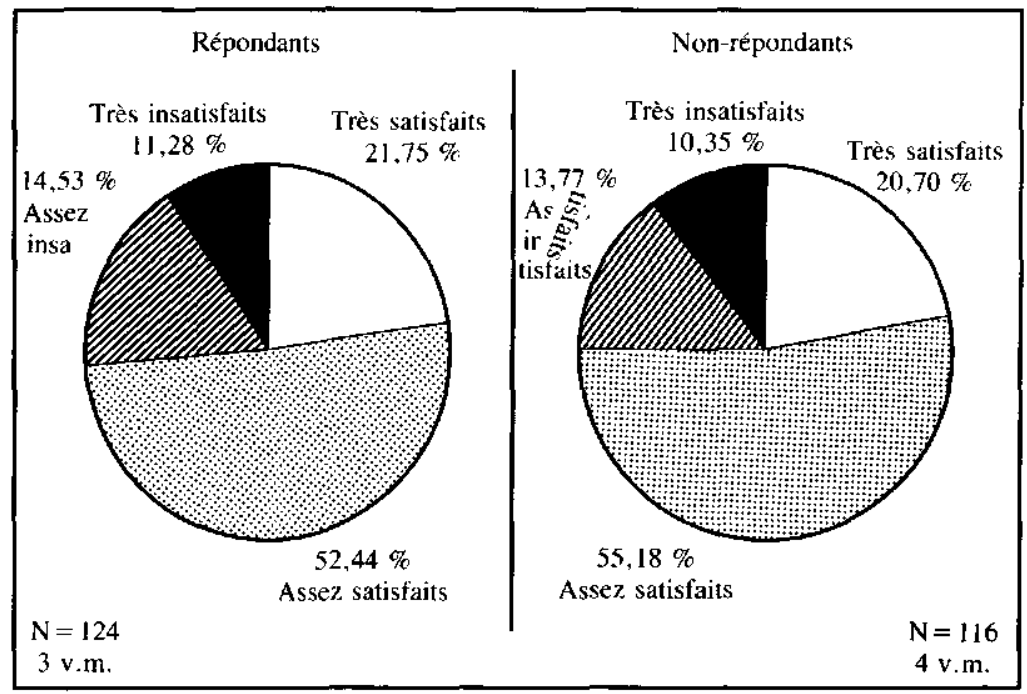


126 répondants à la déclaration $(10,3 \%)$ ont dit en début de sondage ne pas se souvenir d'avoir rempli un tel document, tandis que 52 des 121 nonrépondants $(43 \%)$ disaient ne pas se rappeler avoir été invités à faire une déclaration. Les autres en avaient souvenance, mais pas toujours assez pour répondre à toutes les questions; de là, les nombres de sujets sur lesquels reposent nos pourcentages sont moins élevés que prévu et varient légèrement d'une question à l'autre.

Les personnes qui se souviennent d'avoir répondu à la déclaration ont trouvé l'opération très facile ou facile à $91,7 \%$, contre $7,3 \%$ qui l'ont trouvée assez difficile et $0,9 \%$ très difficile. Les trois quarts $(76,8 \%)$ l'ont remplie sans aide. Plus des quatre cinquièmes $(83,5 \%)$ étaient d'avis que le questionnaire leur permettrait de faire connaître à la cour toutes les conséquences que le crime avait eues sur eux. Quelques-uns auraient voulu un questionnaire plus précis et détaillé $(6,4 \%)$ ou ayant plus de place pour les explications $(4,6 \%)^{6}$. Rappelons ici que, chez les non-répondants, $31,1 \%$ disaient avoir trouvé le questionnaire inadéquat, qu'ils en faisaient même un motif pour ne l'avoir pas complété. Une fois qu'ils ont retoumé leur déclaration, des répondants ont regretté de ne l'avoir pas remplie un peu différemment : ils auraient aimé y changer $(5,3 \%)$, y rajouter $(16,8 \%)$ ou y retrancher $(1,8 \%)$ quelque chose.

Parmi les personnes qui ont complété une déclaration, $54(43,2 \%)$ croient que cette dernière a été utilisée au cours du processus judiciaire, $32(25,6 \%)$ estiment qu'elle ne l'a pas été et $39(31,2 \%)$ l'ignorent.

En fin d'entrevue téléphonique, les sondeurs ont demandé aux sujets si, dans l'éventualité où une personne de leur entourage serait victime d'acte criminel, ils lui recommanderaient de remplir une déclaration de la victime. Tout en plaçant les sujets devant une situation hypothétique, nous voulions voir, dans leurs réponses, un indice de leur propre opinion sur la valeur de la déclaration de la victime. Mises à part vingt personnes qui n'ont pas jugé bon de répondre, $85,7 \%$ des répondants à la déclaration et $82,4 \%$ des nonrépondants disent que oui tandis que $9,2 \%$ et $9,3 \%$ respectivement disent que non et que quelques-uns sont indécis.

Les réponses à cette question sont donc très massivement favorables, chez les non-répondants comme chez les répondants. De la part des premiers, le résultat est frappant puisqu'une réponse favorable est un peu contradictoire avec le fait qu'on n'ait pas soi-même donné suite à la sollicitation. II ne faudrait pas minimiser l'effet de la démarche de recherche elle-même sur ce résultat: qu'on vienne, un an plus tard, vous interroger sur toutes les facettes de la

6. Restent $5,5 \%$ des réponses dont la pertinence nous échappe. 
déclaration de la victime lui confère certainement une importance que bien des personnes ne lui auraient pas reconnue autrement.

\section{LES OPINIONS DES PROFESSIONNELS DE LA JUSTICE SUR LA DÉCLARATION}

Les opinions des professionnels de la justice ont été appréhendées au moyen de 30 entrevues semi-structurées avec des policiers, procureurs de la Couronne, avocats de la défense et juges. Elles ont été analysées qualitativement, de sorte que ses résultats ne peuvent pas être présentés sommairement ici. Néanmoins, je me permets d'énoncer quelques remarques tirées de ces analyses.

Tous les professionnels de la justice sont d'avis que les victimes d'actes criminels doivent faire l'objet de plus d'égards que par le passé et que la déclaration de la victime peut y contribuer. Cette dernière est également vue par tous comme une source d'information nouvelle, dans la mesure bien entendu où ils y ont accès. Or, bien des avocats et des juges ont l'impression que les procureurs en font peu usage. Rappelons à cet égard que souvent les procureurs ne précisent pas de quelle source ils tirent ce dont ils parlent en cour.

Presque personne n'a contesté que les conséquences d'une infraction ou d'un acte criminel sur la victime doivent être prises en compte dans la modulation des sentences, dans la mesure où elles permettent de qualifier plus précisément l'acte commis. Mais il n'y a pas le même consensus sur la pertinence de tenir compte des impacts plus liés aux idiosyncrasies des victimes, et plus particulièrement des impacts psychologiques. Il reste que, pour tous les acteurs de la scène judiciaire, les impacts sur la victime ne sauraient être considérés que comme un élément parmi bien d'autres dans la détermination de la peine. La déclaration de la victime est assez bien admise comme preuve de ces impacts, dans la mesure où elle reste soumise aux règles ordinaires de preuve, mais des avocats de la défense regrettent de devoir porter l'odieux de la contester quand elle s'avère trop dangereuse pour leurs clients.

Quant à la question de savoir si la déclaration de la victime a eu, dans les situations réelles, un effet sur les sentences, elle demeure bien souvent sans réponse tranchée, les juges eux-mêmes étant incapables d'affirmer qu'en l'absence d'une information - qu'ils ont eue en réalité — ils auraient prononcé une sentence identique ou différente.

\section{CONCLUSIONS}

Le projet pilote de déclaration de la victime implanté dans le district judiciaire de Montréal fut d'abord et avant tout une invitation, non insistante, lancée à toutes les victimes de crimes contre la personne et d'introductions 
par effraction, à participer au débat judiciaire. Cette invitation en elle-même constitue une amélioration majeure en termes d'égards pour les victimes.

Près de la moitié des victimes rejointes ont répondu à l'invitation, souvent après avoir hésité, même si c'était plus facile de s'en abstenir. Un quart n'y a pas trouvé d'intérêt. Quant au dernier quart, nos résultats indiquent que, si l'intérêt n'a pas manqué, d'autres considérations ont pris le dessus, la plus importante étant la peur des représailles de l'accusé. Leur choix est à respecter.

Les victimes ont-elles, par leur déclaration, pris davantage la place qui leur revenait dans le débat judiciaire? Sauf exception, leur déclaration a au moins été lue par les procureurs de la Couronne qui y ont puisé de l'information nouvelle pour eux dans $44 \%$ des cas. À partir de là, l'utilisation des déclarations était presque entièrement laissée à la discrétion de la Couronne. Or, les procureurs ont dit s'en être servi une fois sur quatre lors des négociations de plaidoyers ou de sentences avec la défense et une fois sur deux dans leurs plaidoiries devant le juge. De plus, au moins une déclaration sur dix a pesé dans leur décision de faire témoigner ou non la victime.

La défense n'avait qu'un rôle réactif vis-à-vis la déclaration. Elle n'en a certes pas abusé. Quant aux juges, si on a porté à leur connaissance des renseignements tirés de la moitié des déclarations, les procureurs ont compris que ces renseignements avaient compté dans la détermination de la sentence pour un peu moins d'un tiers des causes.

À partir de ces chiffres, notre impression est que la déclaration de la victime a occupé une place tout à fait raisonnable dans le processus judiciaire, compte tenu surtout de sa nouveauté. Par ailleurs, il est certain que la discrétion laissée aux procureurs dans l'usage des déclarations a fait que bon nombre de ces demières n'ont pas été utilisées tout à fait comme leur auteur l'aurait voulu. Les procureurs y ont puisé ce qui leur semblait utile ou acceptable devant la cour. Faut-il le regretter? Il nous semble que non. En effet, dans notre système pénal, les victimes ne sont pas représentées par des avocats et s'il n'est pas question que les procureurs de la couronne deviennent les avocats de la partie lésée, il nous semble néanmoins que la plupart d'entre eux s'insurgent moins contre l'idée de tenir compte des intérêts des victimes. La discrétion qui leur est laissée dans l'utilisation de la déclaration va sûrement dans ce sens. Si, par la diffusion intégrale de leur déclaration, il arrivait aux victimes de se mettre en travers de la démarche poursuivie par les procureurs ou de l'affaiblir, un retour en arrière serait prévisible, de même qu'un accroissement du fardeau qui pèse sur les victimes. C'est le contraire qui est souhaité, d'où nous privilégions, dans l'intérêt des victimes elles-même, que soit maintenue la discrétion des procureurs de la Couronne quant à l'usage de leur déclaration en cour. 
Au terme du projet expérimental, le ministère de la Justice du Québec a décidé de la continuation du programme de déclaration de la victime au Palais de Justice de Montréal. Qui plus est, une équipe travaille actuellement à en étendre la portée aux victimes d'autres types de délits ainsi qu'à d'autres districts judiciaires. Ceci, combiné aux amendements imminents du code criminel favorisant les mesures de réparation à l'endroit des victimes, constitue un pas important vers une véritable reconnaissance de la victime comme sujet de droit. 


\section{ANNEXE \\ DÉCLARATION DE LA VICTIME}

DOSSIER:

La Reine vs

No:

NOM DE LA VICTIME:

DATE DE L'ÉVÉNEMENT:

ACCUSATIONS :

Cette déclaration permettra au juge et aux avocats qui s'occuperont de ce dossier de savoir de quelle façon vous avez été affecté(e) par ce crime.

1. Blessures physiques

A. Avez-vous été blessé(e) lors de l'acte criminel? Si oui, décrivez la nature des blessures.

Oui $\square$ Non

B. Avez-vous eu besoin de soins médicaux? $\mathrm{Si}$ oui, décrivez les traitements que vous avez reçus.

Oui $\square \quad$ Non $\square$

C. À quel endroit (hôpital, clinique, etc.) et pour quelle période de temps avez-vous reçu ces traitements?

D. Avez-vous toujours besoin de traitements (médicaments, physiothérapie, etc.)?

E. Souffrez-vous encore d'une incapacité quelconque (vue, ouïe, membre, prothèse, etc.)?

F. Souffrez-vous ou avez-vous souffert d'autres problèmes de santé, à la suite de l'événement? 
2. Conséquences phychologiques

A. Souffrez-vous ou avez-vous souffert d'un traumatisme émotif ou psychologique depuis l'acte criminel (perte de mémoire, insomnie, etc.)?

B. Cet acte criminet a-t-il eu un impact sur votre vie personnelle ou sur celle de votre entourage (peur, changement de comportement, etc.)?

\section{A. Pertes financières}

Avez-vous subi des pertes financières du fait de l'acte criminel Oui $\square$ Non $\square$

Si ouj, pouvez-vous indiquer la nature et le montant des pertes encourues?

— pertes de salaire: nombre de semaines

montant des pertes

- pertes de revenus: nature

montant des pertes

— objets volés ou endommagés: nature

Montant des pertes

- mesures de sécurité additionnelles (serrure, système d'alarme):

nature

coût

— déménagement: coût

-n soins médicaux: nature

coût

- autres pertes financières

B. Avez-vous été remboursé(e) par vos assurances en totalité ou en partie? 
4. Autres commentaires

Avez-vous d'autres commentaires à ajouter à titre d'information pour le juge et les avocats?

Cette déclaration est faite au meilleur de ma connaissance.

Signature de la victime

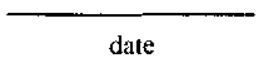

No de téléphone:

Cette déclaration a été remplie avec l'aide de:

Nom

organisme 